\title{
Preoperative lymphocyte to monocyte ratio and prognostic nutritional index were correlated with clinical outcomes in patients with operable colon cancer
}

zhigui li ( $\square$ lizhigui07@sina.com )

West China Hospital, Sichuan University, China https://orcid.org/0000-0002-0936-1131

Tiangen $\mathrm{Ni}$

the second affiliated hospital of chongqing medical university

\section{Yong Zhou}

Sichuan University West China Hospital

Xiaoting Wu

Sichuan University West China Hospital

\section{Research article}

Keywords: colon cancer, lymphocyte to monocyte ratio, prognostic nutritional index, prognosis, systemic inflammation

Posted Date: October 24th, 2019

DOI: https://doi.org/10.21203/rs.2.16440/v1

License: () (1) This work is licensed under a Creative Commons Attribution 4.0 International License. Read Full License 


\section{Abstract}

Background: Systemic inflammation plays an important part in tumorigenesis and progression. The predictive values of the preoperative lymphocyte to monocyte ratio (LMR) and prognostic nutritional index (PNI) in colon cancer remained unclear.

Methods: A total of 308 patients with colon cancer undergoing radical resection were enrolled and analyzed. The receiver operating curves were applied to identify the thresholds for these biomarkers. Kaplan-Meier method and multivariate analysis were used to identify independent prognostic factors.

Results: The univariate analysis showed that elevated LMR and PNI were significantly correlated with better overall survival and progression-free survival. The multivariate analysis showed that LMR and PNI were the independent prognostic factors for overall survival.

Conclusions: Preoperative LMR and PNI could serve as useful prognostic factor in patients with colon cancer undergoing radical resection.

\section{Introduction}

With increasing incidence and mortality, colon cancer represented one of the most frequent cancer and the major cause of cancer-related mortality in the world[1, 2]. Even though the great progress in surgical techniques and adjuvant chemotherapy in recent decades, the 5-year overall survival (OS) of colon cancer was still dissatisfactory in that a large proportion of patients with colon cancer would develop recurrent or metastatic diseases. Some parameters, such as the clinical stage, tumor differentiation, neural invasion, vascular invasion, could predict the prognosis of colon cancer[3]. Neveretheless, the prognosis of patients with the same parameter differed from individul to individual. Meanwhile, it was expensive, time consuming and inconvenient to detect these parameters. There was growing interest in the discovery of prognostic biomarkers contributing to optimize colon cancer management decision-making and improve clinical outcomes.

Systemic inflammation has been proved to play an important part in the tumorigenesis and tumor progression because of inflammatory mediators having the ability of accelerating vascular permeability, facilitating tumor cell infiltration via blood and lymphatic vessels[4-6]. Some biomarkers of peripheral blood examination, such as platelet to lymphocyte ratio (PLR) [7, 8], neutrophil to lymphocyte ratio (NLR) [912], the Glasgow prognostic score (GPS)[13], prognostic nutritional index (PNI)[14], have been shown to reflect the degree of systemic inflammation. This theory has led to widely investigate peripheral blood examination in the hope of developing reliable, reproducible and low-cost prognostic factor. Among these biomarkers, PNI has been reported to be an independent prognostic factor in breast cancer, non-small cell lung cancer $[14,15]$. Peripheral lymphocyte to monocyte ratio (LMR), a new parameter of prognosis, has been drawing more and more attention[16-18]. Up to now, several studies have demonstrated that LMR and PNI were correlated with clinical outcomes in various malignancies, rare studies focused on colon cancer. Proof for the predictive values of LMR and PNI in patients with colon cancer undergoing radical resection remained limited. 
Given all this, the present study was designed to investigate the predictive values of preoperative LMR and PNI in patients with operable colon cancer. These results would facilitate further clinical research on systemic inflammation and optimize the treatment for colon cancer.

\section{Methods}

\section{Patients}

In the present retrospective study, all patients undergoing radical hemicolectomy were recruited at the department of gastrointestinal surgery, West China Hospital between February 2009 and March 2013. The study complied with the principles of the Declaration of Helsinki and was approved by the Ethics Committee of West China Hospital of Sichuan University. The inclusion criteria included: (1) all patients with histologically confirmed colon cancer; (2) age 19-94 years; (3) without distant metastasis; (4) adequate bone marrow function (leukocyte count ranging from $4 \times 10^{9} / \mathrm{L}$ to $10 \times 10^{9} / \mathrm{L}$ ); (5) survival time beyond three months. The exclusion criteria were as follows: (1) patients with inflammatory disease; (2)received preoperative therapy (chemotherapy or radiotherapy), or underwent emergency resection for perforation/obstruction; (3) patients with recurrent or multifocal lesions; (4) missing preoperative blood examination; (5) with other active malignancy; (6) follow up period not exceeding three months.

\section{Peripheral blood parameters and patients' characteristics}

Peripheral blood parameters including absolute lymphocyte count, absolute monocyte count and serum albumin level, were obtained within one week before resection. The LMR was calculated as peripheral absolute lymphocyte count divided by peripheral absolute monocyte count. The PNI was calculated as $0.05 \times$ peripheral absolute lymphocyte count $\left(10^{9} / \mathrm{L}\right)+$ serum albumin levels $(\mathrm{g} / \mathrm{L})$. Patients' clinicopathological features, such as age, gender, the TNM stage, tumor differentiation, etc, were retrieved from the medical database. Follow-up visit was regularly performed until October 2017 or death. The definition of the scoring system were as follow: patient with $L M R \geq 3$ and $P N I \geq 44.2$ was score 0 , patient with $L M R<3$ or $P N I<44.2$ was score 1 , patient with $L M R<3$ and $P N \mid<44.2$ was score 2 .

\section{Statistical analysis}

The follow-up period was defined as our previous study[19]. Progression-free survival (PFS) was calculated from the date of resection to the date of metastasis or recurrence. OS was from the date of surgery to the date of last follow-up or death by any causes. We performed the receiver operating curve (ROC) analysis to count the area under ROC curve (AUC) and then identify the optimal cut-off values of the LMR and PNI. The average was presented as mean \pm standard deviation or median (quartile range). The relationships among the LMR, PNI and clinicopathological features were analyzed by the chisquare test or Fisher exact test. The Kaplan-Meier method with a log-rank test was employed to perform univariate analysis. Independent prognostic factors for OS and PFS were identified by using multivariate Cox-regression analysis with a 
forward conditional method. SPSS 19.0 software (SPSS, Inc., Chicago, IL, USA) was employed to perform all statistical analyses. We considered thevalue of two-tailed $P<0.05$ as statistically significant.

\section{Results}

A cohort of 364 patients with colon cancer were screened in the present study. Fifty-six of them were excluded because of distant metastasis $(n=39)$, multifocal lesions $(n=6)$, perioperative mortality $(n=1)$, received preoperative therapy $(n=3)$, loss to follow up $(n=7)$. After exclusion, 308 patients with stage I-III colon cancer were enrolled in the present study. Details about baseline patient characteristics and clinicopathological features were shown in Table 1. Of these, there were 173 males, and 135 females, 127 patients were under 60 years old, 181 patients were over 60 years old. The median age at the time of diagnosis was 62 years (range 19-94). The median follow-up period from resection was 49 months (range 4-69). The median value of PNI was 45.9 (42.0-49.8); LMR was 4.08 (3.16-5.77). During the follow-up period, $46(14.9 \%)$ out of 308 patients developed metastatic disease, $40(13.0 \%)$ were dead.

ROC analysis was performed to identify the optimal cutoff values of the LMR and PNI. The AUC in LMR and PNI for OS was $0.640(P=0.004), 0.651(P=0.002)$, respectively (Figure 1$)$. The AUC in LMR and PNI for PFS was $0.595(P=0.040), 0.604(P=0.025)$, respectively (Figure 2). By using ROC analysis, the Youden indexes of LMR and PNI were 3.0 and 44.2 for OS, respectively.

According to the cutoff value of 3 in LMR, all patients were dichotomized into two groups: the low LMR group $(L M R<3.0)$ and the elevated LMR group (LMR $\geq 3.0)$. The 1, 3, 5-year OS rates in the low LMR group were $94.2 \%, 80.6 \%, 74.6 \%$, respectively; and in the elevated LMR group were $98.3 \%, 94.6 \%, 90.5 \%$, respectively (Figure 3A). During the follow-up period, 17 (25.4\%) were dead in the low LMR group; 23 (9.5\%) were dead in the elevated LMR group (log-rank test, $P<0.001$ ). The 1, 3, 5-year PFS rates in the low LMR group were $91.0 \%, 76.1 \%, 76.1 \%$, respectively; and in the elevated LMR group were $95.4 \%, 89.6 \%, 87.6 \%$, respectively (Figure 3B). During the follow-up period, 16 (23.9\%) out of 67 patients in the low LMR group were identified as recurrence or metastasis, $30(12.4 \%)$ out of 241 patients in the elevated LMR group developed recurrent or metastatic disease (log-rank test, $P=0.009$ ).

According to the cutoff value of 44.2 in PNI, all patients were dichotomized into two groups: the low PNI group (PNI <44.2) and the elevated PNI group (PNI $\geq 44.2)$. The 1, 3, 5-year OS rates in the low PNI group were $94.9 \%, 89.8 \%, 78.8 \%$, respectively; and in the high PNI group were $98.9 \%, 95.8 \%, 92.1 \%$, respectively (Figure 3C). During the follow-up period, 25 (21.2\%) were dead in the low PNI group; 15 (7.9\%) were dead in the high PNI group (log-rank test, $P<0.001$ ). The 1, 3, 5-year PFS rates in the low PNI group were 91.5\%, $78.8 \%, 78.8 \%$, respectively; and in the elevated PNI group were $96.3 \%, 91.1 \%, 88.9 \%$, respectively (Figure 3D). During the follow-up period, 25 (21.2\%) out of 118 patients in the low PNI group were identified as recurrence or metastasis, 21 (11.1\%) out of 190 patients in the high PNI group developed recurrent or metastatic disease (log-rank test, $P=0.009)$.

According to the scoring system, all patients were divided into three groups: 78 patients $(25.3 \%)$ in the score 0 group, 164 patients (53.3\%) in the score 1 group, 66 patients (21.4\%) in the score 2 group. The 5 -year OS 
rates in the three groups were $96.2 \%, 87.8 \%, 74.2 \%$, respectively (Figure 3E; log-rank test, $P<0.001$ ). The 5year PFS rates in the three groups were $93.6 \%, 84.8 \%, 75.8 \%$, respectively (Figure 3F; log-rank test, $P<0.001$ ).

We also performed subgroup analysis based on clinical stage to evaluate the predictive values of LMR, PNI and the scoring system. Among patients with stage I-II colon cancer, significant differences were observed in all subgroups (Figure 4A-F). Among patients with stage III colon cancer, significant differences were observed in LMR for OS group and in the scoring system for OS and PFS groups (Figure 5A-F).

The relationship among OS and clinicopathological features were shown in Table 2. In the univariate analysis, tumor location, clinical stage, lymph node involvement, differentiation, diabetes mellitus, LMR, PNI and the scoring system significantly correlated with the OS. Due to clinical stage deriving from distant metastasis, lymph node involvement and tumor depth, only clinical stage entered into the multivariate analysis. Multivariate analysis indicated that tumor location, differentiation, clinical stage, diabetes mellitus, LMR and PNI were independent prognostic factors for OS. The relationship among PFS and clinicopathological features were shown in Table 3. Tumor differentiation, lymph node involvement, clinical stage, diabetes mellitus, LMR, PNI and the scoring system were significantly correlated with the PFS in univariate analysis. Multivariate analysis showed that only tumor differentiation, clinical stage were the independent prognostic factors for PFS.

\section{Discussion}

In the nineteenth century, Rudolph Virchow firstly hypothesized the relationship among systemic inflammation and tumorigenesis. Until recently, cumulative evidences have demonstrated that systemic inflammation correlated with tumorigenesis and tumor progression because inflammatory cells and mediators could facilitate tumor proliferation, angiogenesis and metastasis[4, 20]. Several systemic inflammatory parameters, such as NLR, PLR, GPS, could be used to predict clinical outcomes in various malignancies. LMR and PNI, as the new parameters, have earned great intereset recently[21-23]. The results of these studies concerning the relationship among LMR, PNI and clinical outcomes remained inconsistent. Therefore, we examined the cohort of patients with stage I-III colon cancer to identify the predictive values of LMR and PNI.

In the present study, we have demonstrated that elevated LMR and PNI were significantly correlated with favorable outcomes in operable colon cancer. LMR and PNI were independent predictors of OS among patients with colon cancer undergoing curative hemicolectomy, which were in consistent with previous studies[18, 23]. Some other clinicopathological features, such as tumor location, differentiation, lymph node involvement, clinical stage, diabetes mellitus, still remained significance with OS.

Previous study by Li et al.[10] demonstrated that elevated LMR was significantly correlated with favorable OS and PFS for operable colorectal cancer. In patients who received adjuvant chemotherapy, LMR was independent prognostic factor for OS and PFS. In patients without adjuvant chemotherapy, LMR lost their significances with OS and PFS. The study by Stotz et al.[24] examined a cohort of stage II-III colon cancer to identify the predictive value of LMR, which have shown that LMR was significantly correlated with OS and 
PFS. For subgroup analysis, there was significant relationship in patients with stage III colon cancer, no significant relationship in stage II colon cancer. Another study by Peng et al.[25] was designed to investigate the PNI in stage III colon cancer, which demonstrated that elevated PNI was positively correlated with elevated LMR. Compared with the elevated PNI group, clinical outcomes were dissatisfactory in the low PNI group among the entire study population. There were prominent relationship among LMR and clinical outcomes in stage IIIC colon cancer, but no prominent relationship in stage IIIA-B colon cancer. Another study also investigated the systemic inflammation in patients undergoing potentially curative hemicolectomy for stage I-III colon cancer, and demonstrated that right-sided tumor location was correlated with an elevated SIR[26].

The underlying mechanism of the relationship among the systemic inflammation parameters and clinical outcomes remained elusive. Several hypotheses could contribute to explain the relationship. Lymphocytes and monocytes, the major components of peripheral leukocyte population, could be recruited and migrated from peripheral circulation blood to tumor microenvironment, thereby representing the status of the systemic inflammation and host immune response to tumor burden. Elevated LMR indicated that lymphocytosis and monocytopenia. Previous studies have elucidated that lymphocytes were the essential component of adaptive and innate immunity, contributing to induce cytotoxic cell death and inhibit tumor progression and migration[27-29]. It was hypothesized that peripheral absolute lymphocyte count could reflect the degree of host immune response to tumor burden[30]. Lymphocytopenia marked as the increase in CD8+ suppressor lymphocytes and the decrease in CD4+ helper lymphocytes[31], was considered to be responsible for the weak, insufficient immunologic reaction against tumor, thereby representing the unfavorable prognosis in various malignancies. Lymphocytosis could lead to upregulate the immune response against tumor, thereby indicating the favorable prognosis. Peripheral circulating monocytes were recruited to the tumor microenvironment and differentiated into tumor-associated macrophages (TAMs) which have been classified as M1 or M2 phenotypes. The majority of TAMs have an M2 protumoral phenotype, contributing to promote tumor progression, angiogenesis by releasing some cytokines and chemokines[32]. Increased peripheral monocyte counts could reflect the increased levels of TAMs and lead to upregulate the immunosupression, thereby indicating unfavorable prognosis. Our previous studies also demonstrated that peripheral lymphocytosis and monocytopenia predicted favorable outcomes in colon cancer[19, 33].

Serum albumin produced in the liver was the largest quantity of blood plasma protein, playing an important part in physiological function, such as maintaining the colloid osmotic pressure, transporter of various substances, detoxifier and antioxidant. Several studies demonstrated that malnutrition indicated an increased risk of postoperative morbidity and mortality. The main characteristics of malnutrition were hypoalbuminemia and lymphocytopenia. Thus, serum albumin was one of the most important indicator for nutritional status, which has been proved to be an independent prognostic indicator in various malignancies, such as gastric cancer[34], colorectal cancer[35], breast cancer[36].

It must be acknowledged that the present study have several limitations. First, our study was a retrospective single-center cohort study with a small number of patients. Second, other medical conditions, such as infection, ischemia, postoperative adjuvant chemotherapy, which might potentially affect the clinical 
outcomes or peripheral leukocyte count, were not evaluated in this study. Therefore, selection bias could not be entirely excluded in the study. Third, the cutoff values of LMR and PNI were calculated by the method of Youden index. Our cutoff values were inconsistent with previous study. Overall, further larger prospective studies should be conducted to verify the results. The strength of the study included that the study only recruited operable colon cancer and we comprehensively compared the LMR and PNI.

\section{Conclusions}

The present study demonstrated that preoperative PNI and LMR were independent prognostic factors for OS in patients with stage I-III colon cancer undergoing curative hemicolectomy. Patients with elevated LMR and PNI predicted favorable clinical outcomes. On the basis of LMR and PNI, patients with high risk was required to receive intensive postoperative treatment for preventing recurrence or metastasis.

\section{Abbreviations}

LMR: Lymphocyte to monocyte ratio; PNI: Prognostic nutritional index; OS: Overall survival; PFS:

Progression-free survival; PLR: Platelet to lymphocyte ratio; NLR: Neutrophil to lymphocyte ratio; GPS: Glasgow prognostic score; ROC: Receiver operating curve; AUC: Area under ROC curve; TAMs: Tumorassociated macrophages

\section{Declarations}

\section{Acknowledgements}

Not applicable

\section{Authors' contributions}

Z-GL and T-GN designed the study, gather data and drafted this manuscript. $Y Z$ and $X-T W$ were in charge of interpretation the data and the manuscript revising. All authors have read the manuscript and agreed to submit it for publication.

\section{Funding}

Not applicable

\section{Availability of data and materials}

The datasets analysed during the current study are available from the corresponding author on reasonable request. 


\section{Ethics approval and consent to participate}

The study complied with the principles of the Declaration of Helsinki and was approved by the Ethics Committee of West China Hospital of Sichuan University.

\section{Consent for publication}

Not applicable

\section{Competing interests}

The authors declare that they have no competing interests

\section{Author details}

${ }^{1}$ Department of gastrointestinal surgery, West China Hospital, Sichuan University, Chengdu, Sichuan, China.

${ }^{2}$ Department of Breast and Thyroid Surgery, the Second Affiliated Hospital of Chongqing Medical University, Chongqing, China.

\section{References}

1.Chen W, Zheng R, Baade PD, Zhang S, Zeng H, Bray F, et al. Cancer statistics in China, 2015. CA: a cancer journal for clinicians. 2016;66(2):115-32.

2.Siegel RL, Miller KD, Jemal A. Cancer statistics, 2016. CA: a cancer journal for clinicians. 2016;66(1):7-30.

3.Zlobec I, Lugli A. Prognostic and predictive factors in colorectal cancer. Journal of clinical pathology. 2008;61(5):561-9.

4.Coffelt SB, de Visser KE. Cancer: Inflammation lights the way to metastasis. Nature. 2014;507(7490):489.

5.Rossi S, Basso M, Strippoli A, Schinzari G, D’Argento E, Larocca M, et al. Are Markers of Systemic Inflammation Good Prognostic Indicators in Colorectal Cancer? Clinical colorectal cancer. 2017;16(4):26474.

6.Hanahan D, Weinberg RA. Hallmarks of cancer: the next generation. Cell. 2011;144(5):646-74.

7.Cummings M, Merone L, Keeble C, Burland L, Grzelinski M, Sutton K, et al. Preoperative neutrophil:lymphocyte and platelet:lymphocyte ratios predict endometrial cancer survival. British journal of cancer. 2015;113(2):311-20. 
8.Kim JH, Lee JY, Kim HK, Lee JW, Jung SG, Jung K, et al. Prognostic significance of the neutrophil-tolymphocyte ratio and platelet-to-lymphocyte ratio in patients with stage III and IV colorectal cancer. World journal of gastroenterology. 2017;23(3):505-15.

9.Gemenetzis G, Bagante F, Griffin JF, Rezaee N, Javed AA, Manos LL, et al. Neutrophil-to-lymphocyte Ratio is a Predictive Marker for Invasive Malignancy in Intraductal Papillary Mucinous Neoplasms of the Pancreas. Annals of surgery. 2017;266(2):339-45.

10.Li Y, Jia H, Yu W, Xu Y, Li X, Li Q, et al. Nomograms for predicting prognostic value of inflammatory biomarkers in colorectal cancer patients after radical resection. International journal of cancer. 2016;139(1):220-31.

11.Bagley SJ, Kothari S, Aggarwal C, Bauml JM, Alley EW, Evans TL, et al. Pretreatment neutrophil-tolymphocyte ratio as a marker of outcomes in nivolumab-treated patients with advanced non-small-cell lung cancer. Lung cancer. 2017;106:1-7.

12.Park JH, van Wyk H, Roxburgh CSD, Horgan PG, Edwards J, McMillan DC. Tumour invasiveness, the local and systemic environment and the basis of staging systems in colorectal cancer. British journal of cancer. 2017;116(11):1444-50.

13.Suzuki Y, Okabayashi K, Hasegawa H, Tsuruta M, Shigeta K, Kondo T, et al. Comparison of Preoperative Inflammation-based Prognostic Scores in Patients With Colorectal Cancer. Annals of surgery. 2018;267(3):527-31.

14.Mori S, Usami N, Fukumoto K, Mizuno T, Kuroda H, Sakakura N, et al. The Significance of the Prognostic Nutritional Index in Patients with Completely Resected Non-Small Cell Lung Cancer. PloS one. 2015;10(9):e0136897.

15.Mohri T, Mohri Y, Shigemori T, Takeuchi K, Itoh Y, Kato T. Impact of prognostic nutritional index on longterm outcomes in patients with breast cancer. World journal of surgical oncology. 2016;14(1):170.

16.Yang YT, Jiang JH, Yang HJ, Wu ZJ, Xiao ZM, Xiang BD. The lymphocyte-to-monocyte ratio is a superior predictor of overall survival compared to established biomarkers in HCC patients undergoing liver resection. Sci Rep-Uk. 2018;8.

17.Chan JC, Diakos Cl, Chan DL, Engel A, Pavlakis N, Gill A, et al. A Longitudinal Investigation of Inflammatory Markers in Colorectal Cancer Patients Perioperatively Demonstrates Benefit in Serial Remeasurement. Annals of surgery. 2017.

18.Chan JC, Chan DL, Diakos Cl, Engel A, Pavlakis N, Gill A, et al. The Lymphocyte-to-Monocyte Ratio is a Superior Predictor of Overall Survival in Comparison to Established Biomarkers of Resectable Colorectal Cancer. Annals of surgery. 2017;265(3):539-46.

19.Li ZG, Zhao R, Cui YP, Zhou Y, Wu XT. The dynamic change of neutrophil to lymphocyte ratio can predict clinical outcome in stage I-III colon cancer. Sci Rep-Uk. 2018;8. 
20.Mantovani A, Allavena P, Sica A, Balkwill F. Cancer-related inflammation. Nature. 2008;454(7203):43644.

21.Ma JY, Liu Q. Clinicopathological and prognostic significance of lymphocyte to monocyte ratio in patients with gastric cancer: A meta-analysis. International journal of surgery. 2018;50:67-71.

22.Zhu JY, Liu CC, Wang L, Zhong M, Tang HL, Wang H. Peripheral blood lymphocyte-to-monocyte ratio as a prognostic factor in advanced epithelial ovarian cancer: a multicenter retrospective study. Journal of Cancer. 2017;8(5):737-43.

23.Liu X, Li M, Zhao F, Zhu Y, Luo Y, Kong L, et al. The lymphocyte-monocyte ratio predicts tumor response and survival in patients with locally advanced esophageal cancer who received definitive chemoradiotherapy. OncoTargets and therapy. 2017;10:871-7.

24.Stotz M, Pichler M, Absenger G, Szkandera J, Arminger F, Schaberl-Moser R, et al. The preoperative lymphocyte to monocyte ratio predicts clinical outcome in patients with stage III colon cancer. British journal of cancer. 2014;110(2):435-40.

25.Peng J, Zhang R, Zhao Y, Wu X, Chen G, Wan D, et al. Prognostic value of preoperative prognostic nutritional index and its associations with systemic inflammatory response markers in patients with stage III colon cancer. Chinese journal of cancer. 2017;36(1):96.

26.Patel M, McSorley ST, Park JH, Roxburgh CSD, Edwards J, Horgan PG, et al. The relationship between right-sided tumour location, tumour microenvironment, systemic inflammation, adjuvant therapy and survival in patients undergoing surgery for colon and rectal cancer. British journal of cancer. 2018;118(5):705-12.

27.Sadelain M, Riviere I, Riddell S. Therapeutic T cell engineering. Nature. 2017;545(7655):423-31.

28.Dunn GP, Old LJ, Schreiber RD. The immunobiology of cancer immunosurveillance and immunoediting. Immunity. 2004;21(2):137-48.

29.Minami T, Minami T, Shimizu N, Yamamoto Y, De Velasco M, Nozawa M, et al. Identification of Programmed Death Ligand 1-derived Peptides Capable of Inducing Cancer-reactive Cytotoxic $T$ Lymphocytes From HLA-A24+ Patients With Renal Cell Carcinoma. Journal of immunotherapy. 2015;38(7):285-91.

30.Ceze N, Thibault G, Goujon G, Viguier J, Watier H, Dorval E, et al. Pre-treatment lymphopenia as a prognostic biomarker in colorectal cancer patients receiving chemotherapy. Cancer chemotherapy and pharmacology. 2011;68(5):1305-13.

31.Menges T, Engel J, Welters I, Wagner RM, Little S, Ruwoldt R, et al. Changes in blood lymphocyte populations after multiple trauma: association with posttraumatic complications. Critical care medicine. 1999;27(4):733-40. 
32.Marech I, Ammendola M, Sacco R, Sammarco G, Zuccala V, Zizzo N, et al. Tumour-associated macrophages correlate with microvascular bed extension in colorectal cancer patients. Journal of cellular and molecular medicine. 2016;20(7):1373-80.

33.Li Z, Xu Z, Huang Y, Zhao R, Cui Y, Zhou Y, et al. The predictive value and the correlation of peripheral absolute monocyte count, tumor-associated macrophage and microvessel density in patients with colon cancer. Medicine. 2018;97(21):e10759.

34.Toiyama Y, Yasuda H, Ohi M, Yoshiyama S, Araki T, Tanaka K, et al. Clinical impact of preoperative albumin to globulin ratio in gastric cancer patients with curative intent. American journal of surgery. 2017;213(1):120-6.

35.Artac M, Uysal M, Karaagac M, Korkmaz L, Er Z, Guler T, et al. Prognostic Impact of Neutrophil/Lymphocyte Ratio, Platelet Count, CRP, and Albumin Levels in Metastatic Colorectal Cancer Patients Treated with FOLFIRI-Bevacizumab. Journal of gastrointestinal cancer. 2017;48(2):176-80.

36. Hwang KT, Chung JK, Roh EY, Kim J, Oh S, Kim YA, et al. Prognostic Influence of Preoperative Fibrinogen to Albumin Ratio for Breast Cancer. Journal of breast cancer. 2017;20(3):254-63.

\section{Tables}

Table 1. baseline characteristics of patients with colon cancer 


\begin{tabular}{|c|c|c|c|c|c|c|c|c|c|c|c|}
\hline \multirow[t]{2}{*}{ variable } & & \multicolumn{3}{|r|}{ LMR } & \multicolumn{3}{|c|}{ PNI } & \multicolumn{4}{|c|}{ Score } \\
\hline & & $<3$ & $\geq 3$ & $P$ & $<44.2$ & $\geq 44.2$ & $P$ & 0 & 1 & 2 & $P$ \\
\hline \multirow[t]{2}{*}{ Age(years) } & $<60$ & 24 & 103 & 0.309 & 35 & 92 & 0.001 & 77 & 41 & 9 & 0.015 \\
\hline & $\geq 60$ & 43 & 138 & & 83 & 98 & & 86 & 64 & 31 & \\
\hline \multirow[t]{2}{*}{ Gender } & male & 43 & 130 & 0.135 & 66 & 107 & 0.947 & 89 & 59 & 25 & 0.666 \\
\hline & female & 24 & 111 & & 52 & 83 & & 74 & 46 & 15 & \\
\hline \multirow[t]{2}{*}{$\begin{array}{l}\text { Tumor } \\
\text { location }\end{array}$} & $\begin{array}{l}\text { Right } \\
\text { colon }\end{array}$ & 42 & 136 & 0.359 & 78 & 100 & 0.020 & 84 & 68 & 26 & 0.062 \\
\hline & $\begin{array}{l}\text { Left } \\
\text { colon }\end{array}$ & 25 & 105 & & 40 & 90 & & 79 & 37 & 14 & \\
\hline \multirow{2}{*}{$\begin{array}{l}\text { Tumor } \\
\text { invasion } \\
\text { depth }\end{array}$} & $\mathrm{T} 1 \mathrm{~T} 2$ & 3 & 33 & 0.038 & 13 & 23 & 0.773 & 22 & 12 & 2 & 0.324 \\
\hline & $\mathrm{T} 3 \square \mathrm{T} 4$ & 64 & 208 & & 105 & 167 & & 141 & 93 & 38 & \\
\hline \multirow{2}{*}{$\begin{array}{l}\text { Lymph node } \\
\text { metastasis }\end{array}$} & No & 50 & 167 & 0.397 & 78 & 139 & 0.187 & 116 & 74 & 27 & 0.902 \\
\hline & Yes & 17 & 74 & & 40 & 51 & & 47 & 31 & 13 & \\
\hline \multirow[t]{2}{*}{ Tumor stage } & $\mathrm{I}+\mathrm{III}$ & 50 & 167 & 0.397 & 78 & 139 & 0.187 & 116 & 74 & 27 & 0.902 \\
\hline & III & 17 & 74 & & 40 & 51 & & 47 & 31 & 13 & \\
\hline \multirow[t]{2}{*}{ Tumor grade } & low & 24 & 76 & 0.508 & 41 & 59 & 0.501 & 51 & 33 & 16 & 0.552 \\
\hline & $\begin{array}{l}\text { Middle, } \\
\text { high }\end{array}$ & 43 & 165 & & 77 & 131 & & 112 & 72 & 24 & \\
\hline \multirow[t]{2}{*}{ Hypertension } & No & 56 & 196 & 0.629 & 94 & 157 & 0.514 & 135 & 82 & 34 & 0.517 \\
\hline & Yes & 11 & 46 & & 24 & 33 & & 28 & 23 & 6 & \\
\hline \multirow{2}{*}{$\begin{array}{l}\text { Diabetes } \\
\text { mellitus }\end{array}$} & No & 60 & 225 & 0.294 & 110 & 175 & 0.717 & 152 & 96 & 37 & 0.859 \\
\hline & Yes & 7 & 16 & & 8 & 15 & & 11 & 9 & 3 & \\
\hline
\end{tabular}

Table 2. Univariate and multivariate analysis of prognostic factors for overall survival 


\begin{tabular}{|c|c|c|c|c|}
\hline \multicolumn{2}{|l|}{ Variables } & \multirow{3}{*}{$\begin{array}{c}\text { Univariate analysis } \\
P \text { value } \\
0.208\end{array}$} & \multicolumn{2}{|c|}{ Multivariate analysis } \\
\hline & & & HR (95\%CI) & $P$ value \\
\hline Age(years) & $\begin{array}{l}\square 60 \\
\geq 60\end{array}$ & & & \\
\hline Gender & $\begin{array}{l}\text { Male } \\
\text { Female }\end{array}$ & 0.563 & & \\
\hline Tumor location & $\begin{array}{l}\text { Right colon } \\
\text { Left colon }\end{array}$ & 0.036 & $\begin{array}{c}1 \\
0.447(0.219-0.912)\end{array}$ & 0.027 \\
\hline Differentiation & $\begin{array}{l}\text { Low } \\
\text { Middle, high }\end{array}$ & 0.001 & $\begin{array}{c}1 \\
0.432(0.230-0.811)\end{array}$ & 0.009 \\
\hline Tumor invasion depth & $\begin{array}{l}\text { T1 वT2 } \\
\text { T3ロT4 }\end{array}$ & 0.171 & & \\
\hline Lymph node involvement & $\begin{array}{l}\text { No } \\
\text { Yes }\end{array}$ & 0.001 & & \\
\hline Clinical stage & $\begin{array}{l}\text { I } \square \text { II } \\
\text { III }\end{array}$ & 0.001 & $\begin{array}{c}1 \\
2.823(1.488-5.357)\end{array}$ & 0.001 \\
\hline Hypertension & $\begin{array}{l}\text { No } \\
\text { Yes }\end{array}$ & 0.398 & & \\
\hline Diabetes mellitus & $\begin{array}{l}\text { No } \\
\text { Yes }\end{array}$ & 0.008 & $\begin{array}{c}1 \\
3.043(1.305-7.099)\end{array}$ & 0.010 \\
\hline LMR & $\begin{array}{l}\square 3 \\
\geq 3\end{array}$ & 0.000 & $\begin{array}{c}1 \\
0.852(0.731-0.992)\end{array}$ & 0.039 \\
\hline PNI & $\begin{array}{l}\square 44.2 \\
\geq 44.2\end{array}$ & 0.000 & $\begin{array}{c}1 \\
0.415(0.216-0.797)\end{array}$ & 0.008 \\
\hline Score & $\begin{array}{l}0 \\
1 \\
2\end{array}$ & 0.000 & & \\
\hline
\end{tabular}

Table 3. Univariate and multivariate analysis of prognostic factors for progression-free survival

\begin{tabular}{|c|c|c|c|c|}
\hline \multirow{2}{*}{\multicolumn{2}{|c|}{ Variables }} & \multirow{2}{*}{$\frac{\text { Univariate analysis }}{P \text { value }}$} & \multicolumn{2}{|c|}{ Multivariate analysis } \\
\hline & & & HR $(95 \% \mathrm{CI})$ & $P$ value \\
\hline Age(years) & $\begin{array}{l}<60 \\
\geq 60\end{array}$ & 0.101 & & \\
\hline Gender & $\begin{array}{l}\text { Male } \\
\text { Female }\end{array}$ & 0.692 & & \\
\hline Tumor location & $\begin{array}{l}\text { Right colon } \\
\text { Left colon }\end{array}$ & 0.252 & & \\
\hline Differentiation & $\begin{array}{l}\text { Low } \\
\text { Middle, high }\end{array}$ & 0.001 & $\begin{array}{c}1 \\
0.428(0.239-0.767)\end{array}$ & 0.004 \\
\hline Tumor invasion depth & $\begin{array}{l}\text { T1 घT2 } \\
\text { T3ロT4 }\end{array}$ & 0.102 & & \\
\hline Lymph node involvement & $\begin{array}{l}\text { No } \\
\text { Yes }\end{array}$ & 0.000 & & \\
\hline Clinical stage & $\begin{array}{l}\text { I } \square \text { II } \\
\text { III }\end{array}$ & 0.000 & $\begin{array}{c}1 \\
2.789(1.542-5.043)\end{array}$ & 0.001 \\
\hline Hypertension & $\begin{array}{l}\text { No } \\
\text { Yes }\end{array}$ & 0.275 & & \\
\hline Diabetes mellitus & $\begin{array}{l}\text { No } \\
\text { Yes }\end{array}$ & 0.021 & $\begin{array}{c}1 \\
2.230(0.985-5.046)\end{array}$ & 0.054 \\
\hline LMR & $\begin{array}{l}<3 \\
\geq 3\end{array}$ & 0.009 & $\begin{array}{c}1 \\
0.903(0.795-1.026)\end{array}$ & 0.118 \\
\hline PNI & $\begin{array}{l}<44.2 \\
\geq 44.2\end{array}$ & 0.009 & $\begin{array}{c}1 \\
0.565(0.311-1.027)\end{array}$ & 0.061 \\
\hline Score & $\begin{array}{l}0 \\
1 \\
2 \\
\end{array}$ & 0.000 & & \\
\hline
\end{tabular}


Figures

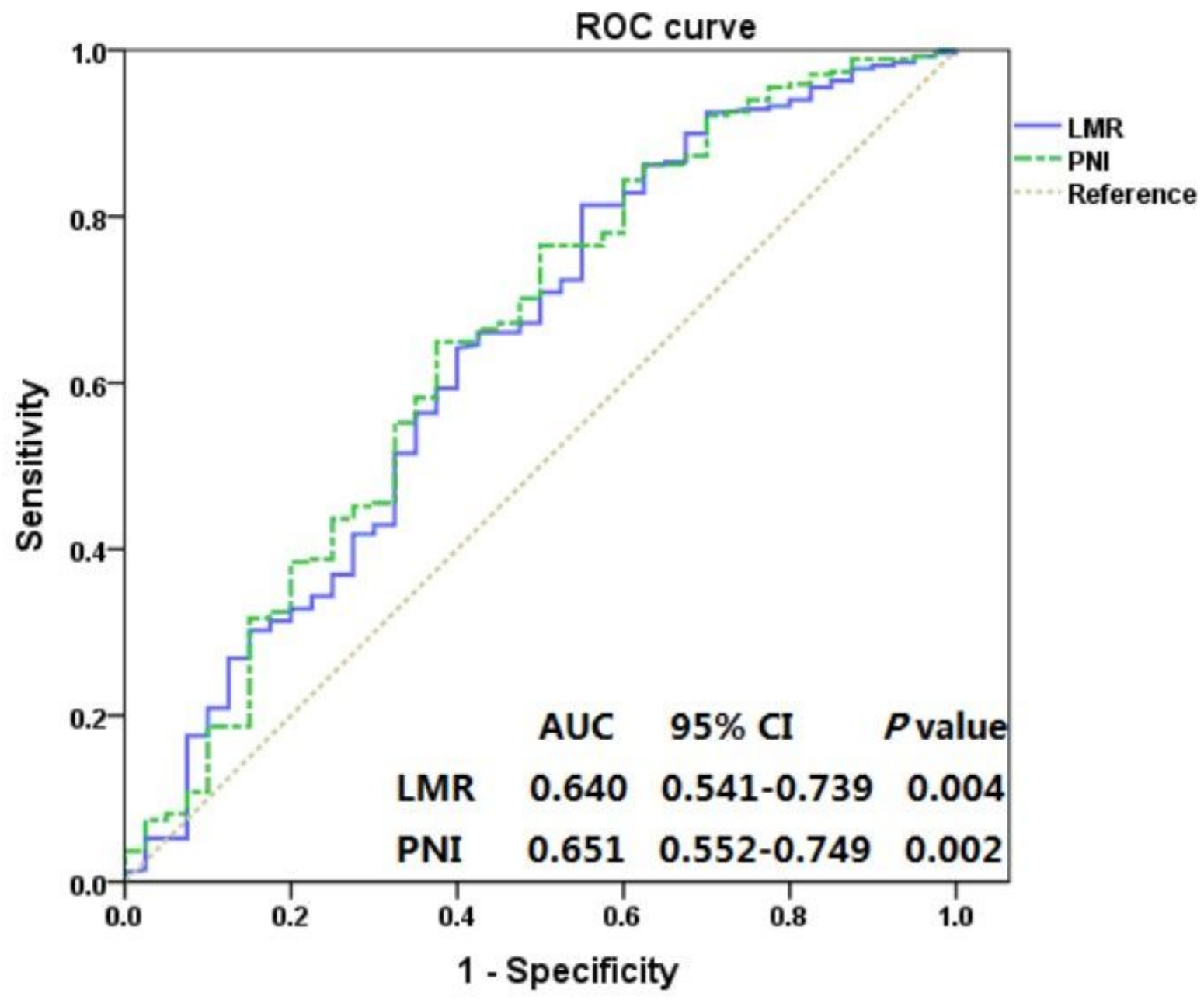

Figure 1

The receiver operating curve analysis of preoperative LMR and PNI according to overall survival. 


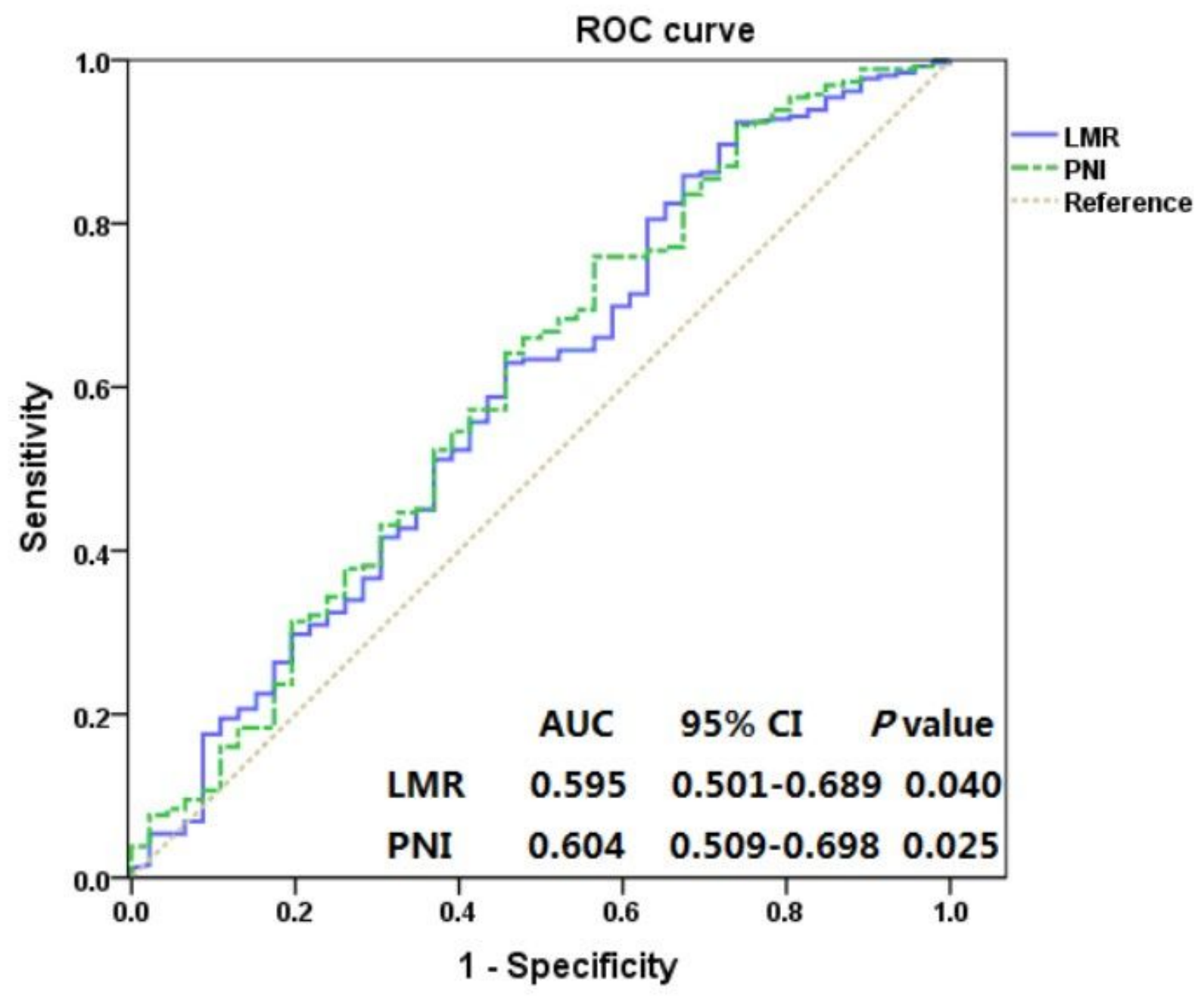

Figure 2

The receiver operating curve analysis of preoperative LMR and PNI according to progression-free survival. 

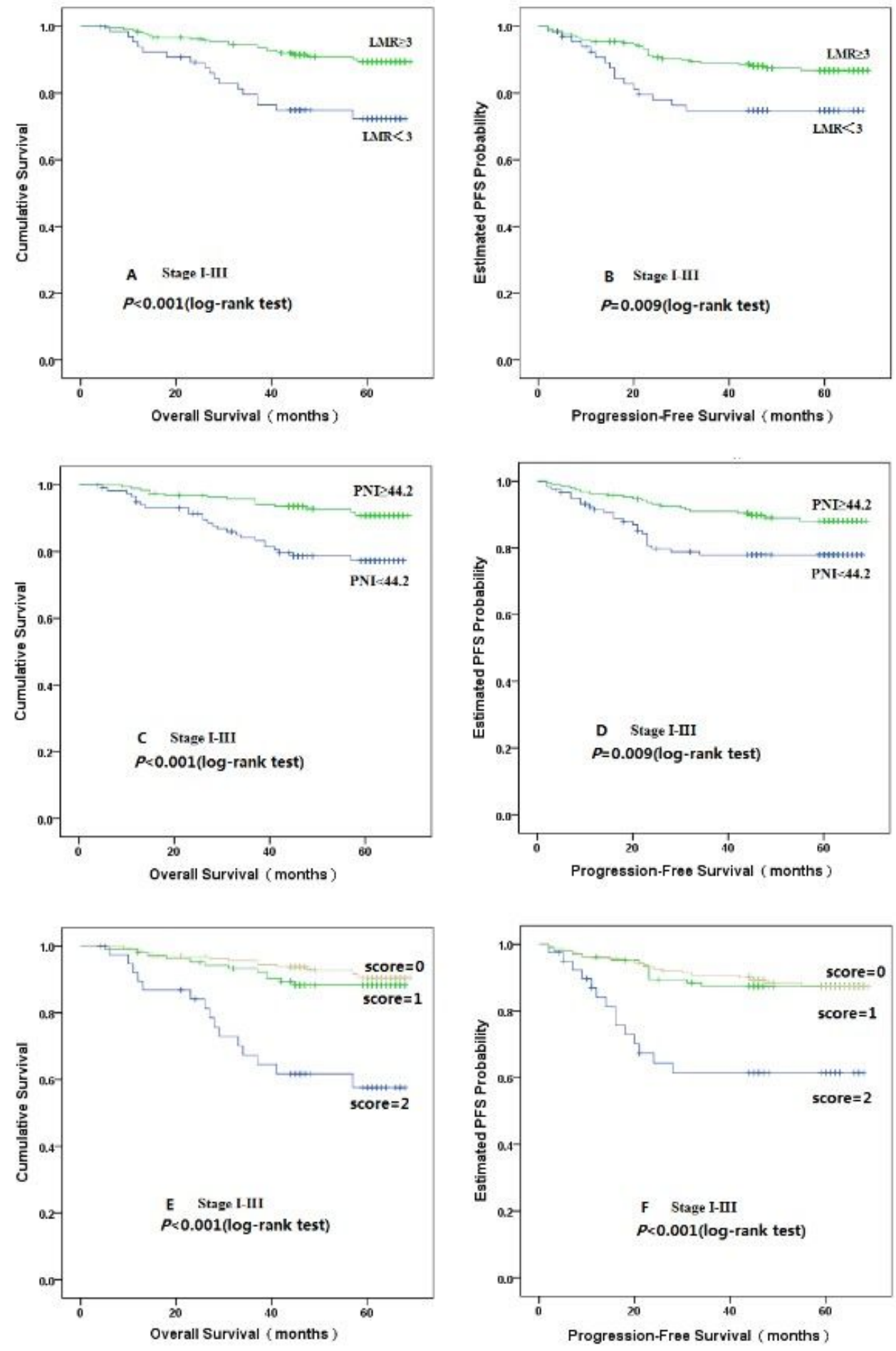

\section{Figure 3}

Kaplan-Meier curves of LMR, PNI and the scoring system for overall survival and progression-free survival in patients with stage I-III colon cancer. Note: A and B are the survival curves of LMR for overall survival and progression-free survival; $C$ and $D$ are the survival curves of $P N I$ for overall survival and progression-free survival; $\mathrm{E}$ and $\mathrm{F}$ are the survival curves of the scoring system for overall survival and progression-free survival. 

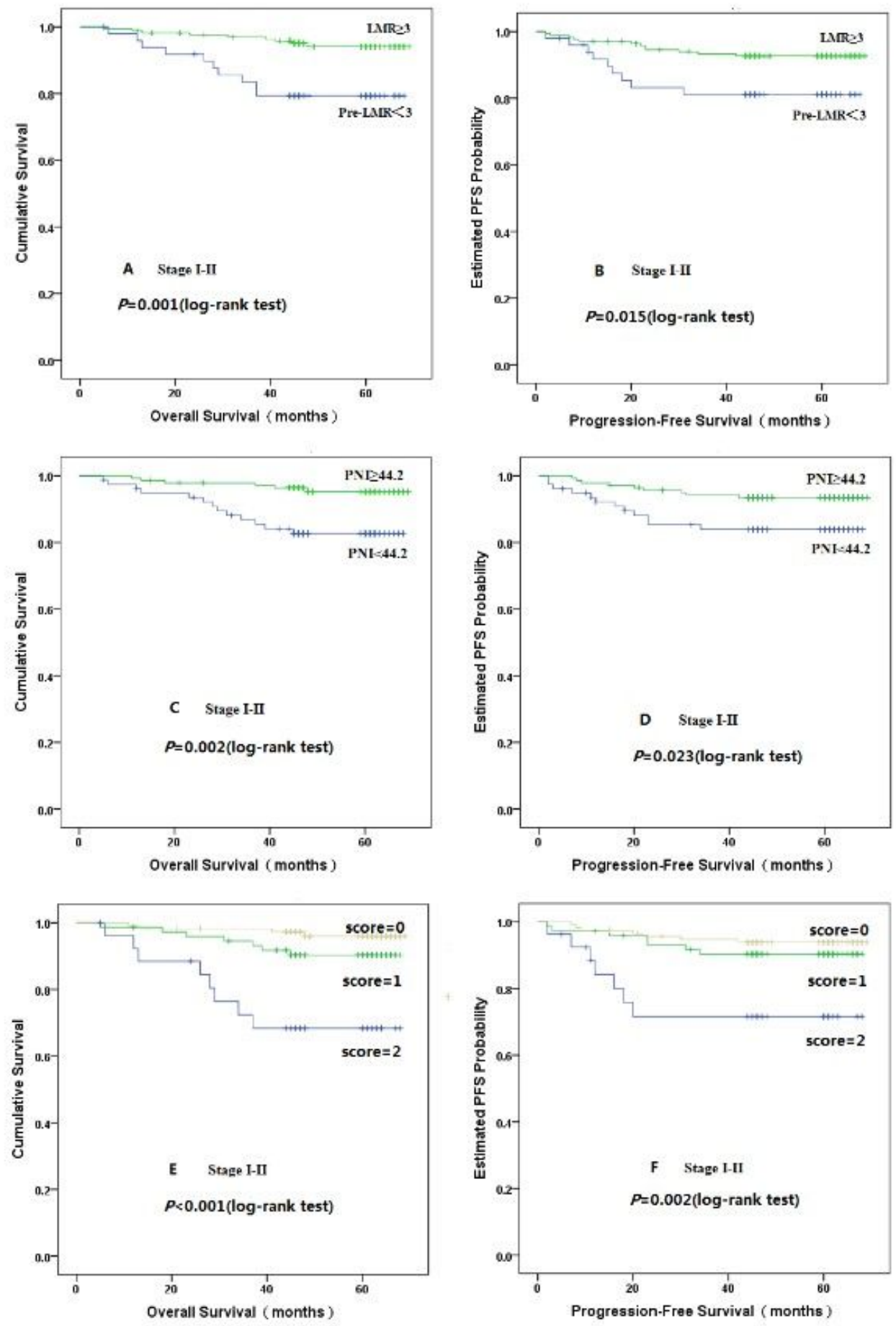

\section{Figure 4}

Kaplan-Meier curves of LMR, PNI and the scoring system for overall survival and progression-free survival in patients with stage I-II colon cancer. Note: A and B are the survival curves of LMR for overall survival and progression-free survival; $C$ and $D$ are the survival curves of PNI for overall survival and progression-free survival; $\mathrm{E}$ and $\mathrm{F}$ are the survival curves of the scoring system for overall survival and progression-free survival. 

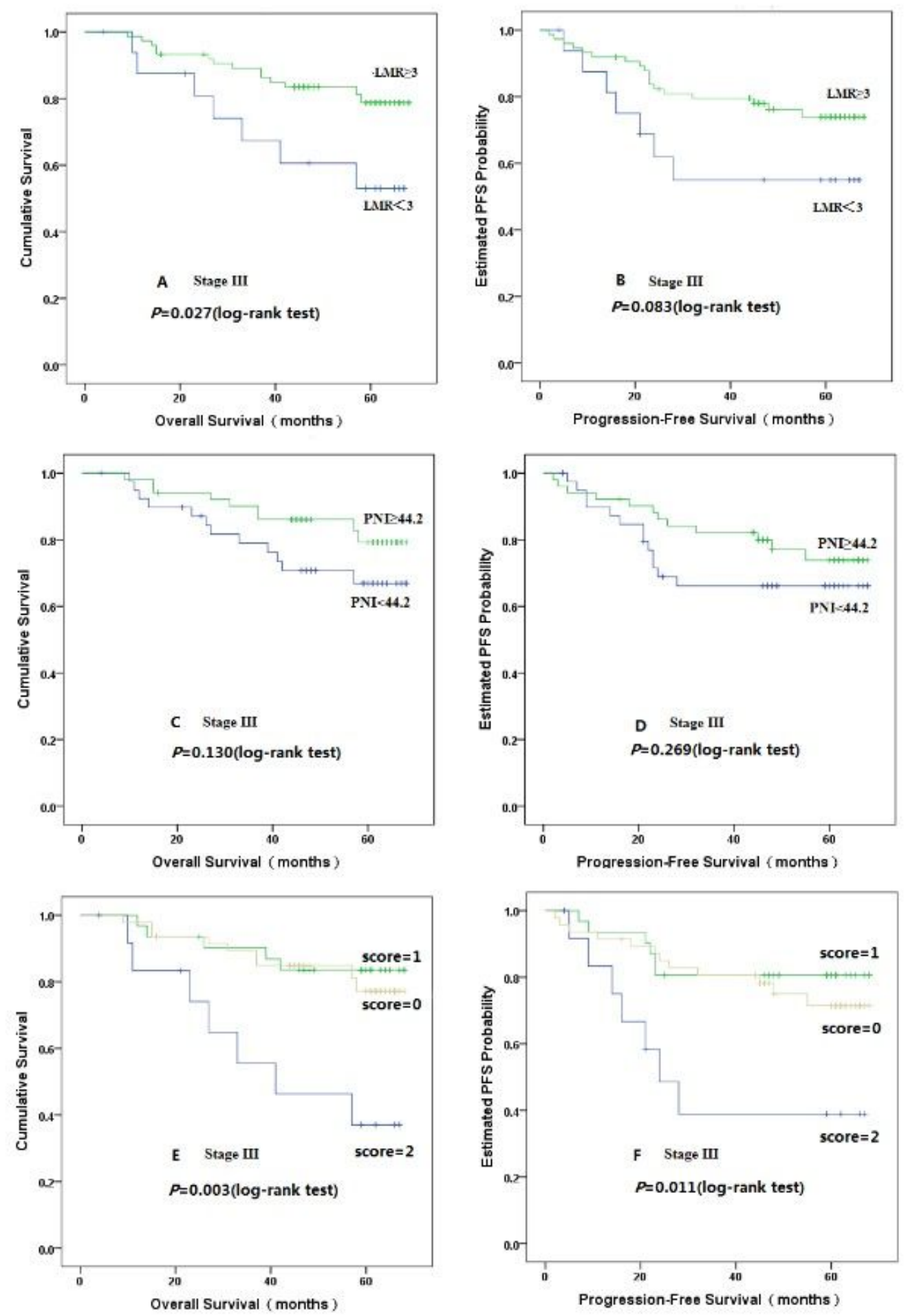

\section{Figure 5}

Kaplan-Meier curves of LMR, PNI and the scoring system for overall survival and progression-free survival in patients with stage III colon cancer. Note: A and B are the survival curves of LMR for overall survival and progression-free survival; $C$ and $D$ are the survival curves of $\mathrm{PNI}$ for overall survival and progression-free survival; $\mathrm{E}$ and $\mathrm{F}$ are the survival curves of the scoring system for overall survival and progression-free survival. 